\title{
miR-133b, a microRNA targeting S1PR1, suppresses nasopharyngeal carcinoma cell proliferation
}

\author{
NAN CHENG and GUANG-HUI WANG \\ Department of Otolaryngology, Huaihe Hospital, Henan University, Kaifeng, Henan 475000, P.R. China
}

Received November 20, 2014; Accepted January 11, 2016

DOI: 10.3892/etm.2016.3043

\begin{abstract}
MicroRNAs (miRs) are a class of short and non-coding RNA molecules, which function as either oncogenes or tumor suppressors in the development of various human cancers, including nasopharyngeal carcinoma (NPC). The aim of the present study was to investigate the expression of miR-133b in NPC tissue samples, as compared with adjacent normal tissues, and to examine its roles and underlying mechanisms. Analysis using reverse transcription-quantitative polymerase chain reaction demonstrated that miR-133b was downregulated in NPC tissue samples, as compared with adjacent tissues. In vitro experiments using NPC cell lines transfected with miR-133b mimics or antisense oligonucleotides further demonstrated that the overexpression of miR-133b mimics impaired, whereas knockdown of its expression promoted, the proliferation of NPC cells. Sphingosine-1-phosphate receptor 1 (S1PR1) was predicted to be a target of miR-133b. Luciferase reporter assays showed that miR-133b inhibited the protein expression of S1PR1 by targeting its 3'-untranslated region. Furthermore, western blot analysis demonstrated that miR-133B altered the regulation of the signal transducer and activator of transcription-3 (STAT3) signaling pathway and the expression of downstream proteins in NPC cells. Therefore, the results of the present study suggested that a previously unknown miR-133b/S1PR1 molecular network may regulate NPC progression.
\end{abstract}

\section{Introduction}

Nasopharyngeal carcinoma (NPC), which has an incidence of 2.8/100,000 per year in men and $1.9 / 100,000$ per year in women, is the most commonly diagnosed head and neck cancer in Southern China (1). NPC has previously been associated with Epstein-Barr virus infection, tobacco consumption

Correspondence to: Dr Nan Cheng, Department of Otolaryngology, Huaihe Hospital, Henan University, 8 Bao-Bei Road, Kaifeng, Henan 475000, P.R. China

E-mail: nanchenghn@yeah.net

Key words: nasopharyngeal carcinoma, sphingosine-1-phosphate receptor 1, microRNA, miR-133b and various genetic alterations (2,3). Furthermore, it has been suggested that microRNAs (miRNAs), which are a class of non-coding RNA molecules, 18-25 nucleotides in length, may have important roles in the carcinogenesis of NPC (4). In previous studies, the expression of several miRNAs, including miR-29c, miR-372, miR-214 and the let-7 family, has been detected in NPC samples and their roles elucidated (5-8). Therefore, regulation of miRNAs may be considered a potential novel strategy for the diagnosis and treatment of patients with NPC.

miR-133b has been identified as a tumor suppressor in numerous types of human cancer (9); miR-133b is often downregulated in gastric cancer and its overexpression has been shown to reduce the metastatic potential of gastric cancer cells $(10,11)$. In addition, miR-133b has been shown to be downregulated in colorectal cancer tissues, as compared with adjacent tissues (12), and has been found to inhibit the proliferation of colon cells by suppressing the TATA box-binding protein-like protein 1 (TBPL1) (12). Furthermore, miR-133b has been shown to inhibit the proliferation, migration and invasion of osteosarcoma cells, and promote their apoptosis (13). In a previous study, serum levels of miR-133b were able to predict the prognosis of patients with osteosarcoma (14). However, to the best of our knowledge, the expression status and clinical significance of miR-133b in NPC have yet to be investigated. The present study aimed to investigate the expression levels of miR-133b in NPC tissue samples, as compared with adjacent normal tissues. Furthermore, its roles and underlying mechanisms, including its effects on cell proliferation, were investigated.

\section{Materials and methods}

Tissue samples. A total of 23 paired NPC and adjacent normal specimens were collected from 23 male patients undergoing routine therapeutic surgery at the Huaihe Hospital (Kaifeng, China) between October 2012 and October 2014. The age range of the patients was 15 to 28 -years-old. Written informed consent was obtained from all patients. All tissue samples were flash-frozen in liquid nitrogen immediately following collection and stored at $-80^{\circ} \mathrm{C}$ until experimentation. The present study was approved by the institutional review board of Huaihe Hospital.

Cell culture. The NPC cell lines, CNE2 and HONE1, were obtained from the Chinese Academy of Sciences Cell Bank 
(Shanghai, China). The cells were cultured in RPMI-1640 medium (Sigma-Aldrich, St. Louis, MO, USA), supplemented with $10 \%$ fetal bovine serum (Merck Millipore, Wanchai, Hong Kong, China). The cultures were maintained at $37^{\circ} \mathrm{C}$ in a humidified atmosphere containing $5 \% \mathrm{CO}_{2}$.

Transient transfection. The CNE2 and HONE1 cells were transfected with miR-133b mimics, miR-133b antisense or negative control (NC; all Genepharm, Inc., Sunnyvale, CA, USA) for $24 \mathrm{~h}$ at room temperature using Lipofactamine 2000 (Invitrogen; Thermo Fisher Scientific, Inc., Inc., Waltham, MA, USA), according to the manufacturer's protocol.

$R N A$ isolation and reverse transcription-quantitative polymerase chain reaction ( $R T-q P C R)$. Total RNA from the patient tissues and CNE2 and HONE1 cell lines was extracted using TRIzol ${ }^{\circledR}$ reagent (Invitrogen; Thermo Fisher Scientific), according to the manufacturer's protocol. The RNA was purified by treatment with PureLink ${ }^{\circledR}$ DNase Set (Thermo Fisher Scientific) and $20 \mu 1$ RNA was reverse transcribed into cDNA using the Reverse Transcription System (Promega Corporation, Madison, WI, USA). The expression levels of hsa-miR-133b were determined using the TaqMan ${ }^{\circledR}$ MicroRNA assay (Applied Biosystems; Thermo Fisher Scientific, Inc.) and the LightCycler ${ }^{\circledR} 480$ instrument (Roche Diagnostics, Basel, Switzerland). The primer sequences were as follows: miR-133b forward, 5'-AAAGGACCCCAACAA CCAGCAA-3' and reverse, 5'-TTGCTGGTTGTTGGGGTC CTTT-3'; and U6 small nuclear (sn)RNA forward, 5'-CTC GCTTCGGCAGCACATATACT-3' and reverse, 5'-ACGCTT CACGAATTTGCGTGTC-3' (Biosune Biotechnology Co., Shanghai, China). U6 snRNA was used as an internal control for normalization and quantification of the miR-133b expression levels using the $2^{-\Delta \Delta C q}$ method (15).

In order to determine the mRNA expression levels of B-cell lymphoma-2 (Bcl-2), myeloid cell leukemia-1 (Mcl-1) and cellular inhibitor of apoptosis-2 (c-IAP2), qPCR was performed using the SYBR Green Master Mix (Thermo Fisher Scientific) and the LightCycler ${ }^{\circledR} 480$ instrument, according to the manufacturer's protocols. The PCR cycling conditions included an initial holding period at $95^{\circ} \mathrm{C}$ for $5 \mathrm{~min}$, followed by 45 cycles of $94^{\circ} \mathrm{C}$ for $5 \mathrm{sec}$ and $60^{\circ} \mathrm{C}$ for $35 \mathrm{sec}$. The primer sequences were as follows: Bcl-2 forward, 5'-GGTGGGGTC ATGTGTGTGG-3' and reverse, 5'-CGGTTCAGGTACTCA GTCATCC-3'; Mcl-1 forward, 5'-TGCTTCGGAAACTGG ACATCA-3' and reverse, 5'-TAGCCACAAAGGCACCAA AAG-3'; c-IAP2 forward, 5'-AAGCTACCTCTCAGCCTA CTTT-3' and reverse, 5'-CCACTGTTTTCTGTACCCGGA-3'; and $\beta$-actin forward, 5'-CATGTACGTTGCTATCCAGGC-3' and reverse, 5'-CTCCTTAATGTCACGCACGAT-3' (Biosune Biotechnology Co.). The $\beta$-actin gene was used as an internal control for normalization and quantification of the relative mRNA expression levels using the $2^{-\Delta \Delta \mathrm{Cq}}$ method (15). The RT-qPCR data were analyzed using the LightCycler ${ }^{\circledR} 480$ software, version 1.5.0 (Roche Diagnostics).

3-(4,5-Dimethylthiazol-2-yl)-2,5-diphenyltetrazolium bromide (MTT) assay. Cell growth was determined using an MTT assay (Sigma-Aldrich). At $24 \mathrm{~h}$ following transfection of the NPC cell lines with miR-133b mimics, miR-133b antisense or NC, the

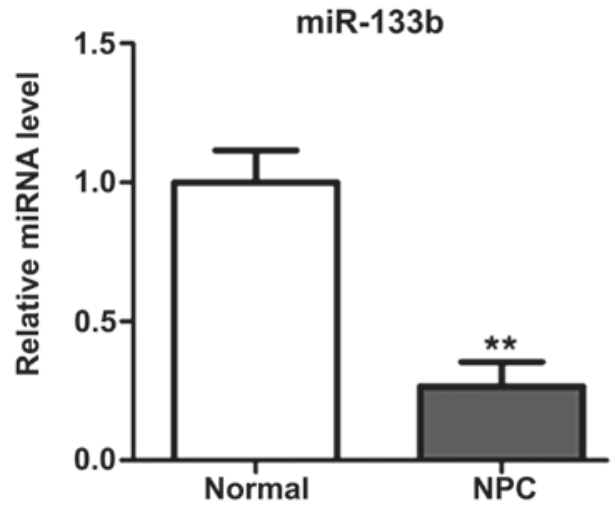

Figure 1. Expression levels of miR-133b in NPC tissues. The expression levels of miR-133b were significantly downregulated in NPC tissue samples, as compared with adjacent normal tissue samples, as determined using reverse transcription-quantitative polymerase chain reaction. ${ }^{* *} \mathrm{P}<0.01$ vs. the normal tissues. miR, microRNA; NPC, nasopharyngeal carcinoma.

cells were seeded into 96 -well plates $\left(2 \times 10^{3}\right.$ cells/well), and cell proliferation was recorded every $24 \mathrm{~h}$ for 4 days. The number of viable cells was assessed by measuring the absorbance at $450 \mathrm{~nm}$ using a microplate reader (Model 3550 Microplate Reader; Bio-Rad Laboratories, Inc., Hercules, CA, USA).

Bromodeoxyuridine (BrdU) assay. A cell proliferation enzyme-linked immunosorbent assay (BrdU kit; Beyotime Institute of Biotechnology, Haimen, China) was used to analyze the incorporation of BrdU into the NPC cell lines during DNA synthesis, according to the manufacturer's protocol. All experiments were performed in triplicate. Absorbance was measured at $450 \mathrm{~nm}$ using the SpectraMax 190 Microplate Reader (Molecular Devices, LLC, Sunnyvale, CA, USA).

$m i R-133 b$ target predictions. The putative targets of miR-133b were predicted using miRWalk software, release 2.0 (http://www.umm.uni-heidelberg.de/apps/zmf/mirwalk/). The algorithm produced a list of target genes for miR-133b by searching for the presence of conserved 7-mer and 8-mer sites matching the seed region of miR-133b.

Western blotting. The NPC cells were harvested and lysed using ice-cold lysis buffer [50 mM Tris-HCl, pH 6.8, 5\% 2-mercaptoethanol, $2 \% \mathrm{w} / \mathrm{v}$ sodium dodecyl sulfate (SDS), $10 \%$ glycerol; Biosune Biotechnology Co.]. Following centrifugation at 20,000 $\mathrm{x}$ g for $10 \mathrm{~min}$ at $4^{\circ} \mathrm{C}$, the proteins in the supernatants were quantified using a Bicinchoninic Acid Assay kit (Pierce Biotechnology Inc., Rockford, IL, USA). The proteins were separated by $10 \%$ SDS-polyacrylamide gel electrophoresis, and transferred to a nitrocellulose membrane (GE Healthcare Life Sciences, Chalfont, UK). After blocking the membrane with $10 \%$ non-fat milk in phosphate-buffered saline, the membranes were incubated overnight at $4^{\circ} \mathrm{C}$ with the following primary antibodies: Rabbit anti-human sphingosine-1-phosphate receptor 1 (S1PR1) polyclonal antibody (1:1,000; sc-25489; Santa Cruz Biotechnology, Inc., Dallas, TX, USA), goat anti-human phosphorylated signal transducer and activator of transcription 3 (p-STAT3) polyclonal antibody (1:500; sc-21876; Santa Cruz Biotechnology), rabbit anti-human total STAT3 polyclonal antibody (1:2,000; sc-482; Santa Cruz Biotechnology) and 


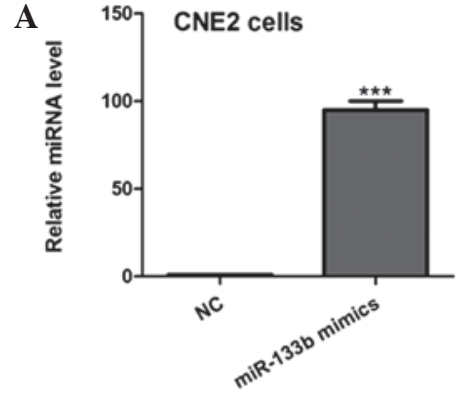

D

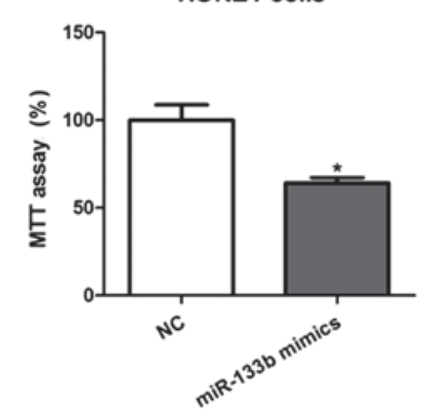

B

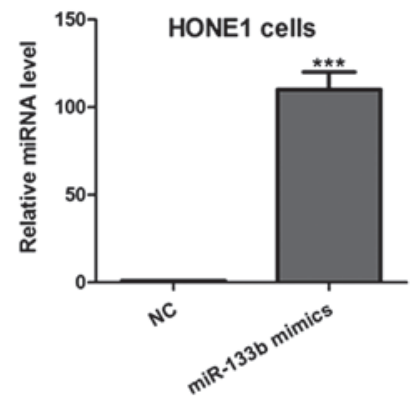

$\mathbf{E}$

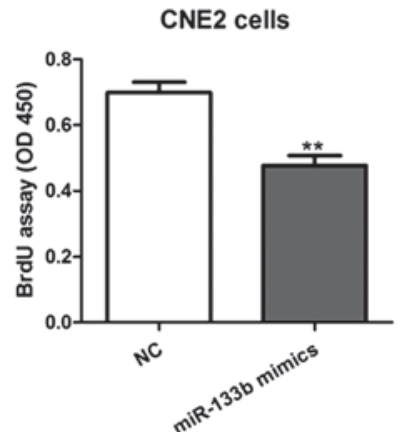

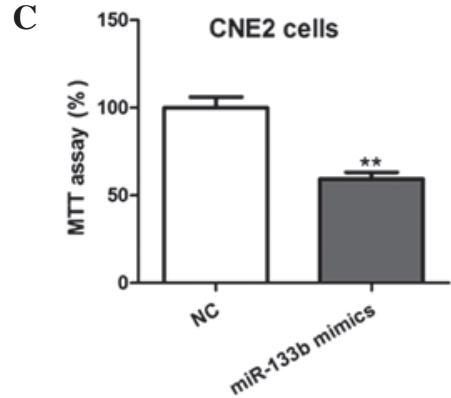

F HONE1 cells

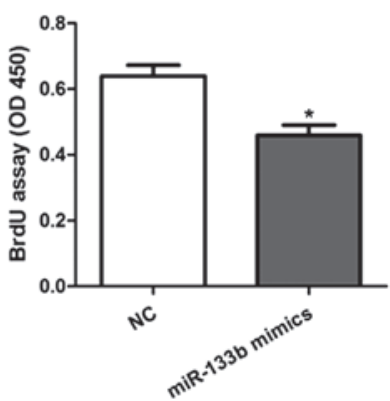

Figure 2. Overexpression of miR-133b inhibited NPC cell proliferation. Expression levels of miR-133b in (A) CNE2 and (B) HONE1 NPC cells following transfection with miR-133b mimics or the $\mathrm{NC}$ were determined using reverse transcription-quantitative polymerase chain reaction. The (C and $\mathrm{D})$ cell growth and (E and F) proliferative potentials of the (C and E) CNE2 and (D and F) HONE1 cells transfected with miR-133b mimics or NC were determined. The optical density at $450 \mathrm{~nm}$ (OD 450) was assayed following transfection for $24 \mathrm{~h}$. $\mathrm{P}<0.05,{ }^{* *} \mathrm{P}<0.01$ and ${ }^{* * * *} \mathrm{P}<0.001 \mathrm{vs}$. the NC. miR, microRNA; NPC, nasopharyngeal carcinoma; NC, normal control; MTT, 3-(4,5-dimethylthiazol-2-yl)-2,5-diphenyltetrazolium bromide; BrdU, bromodeoxyuridine.

A

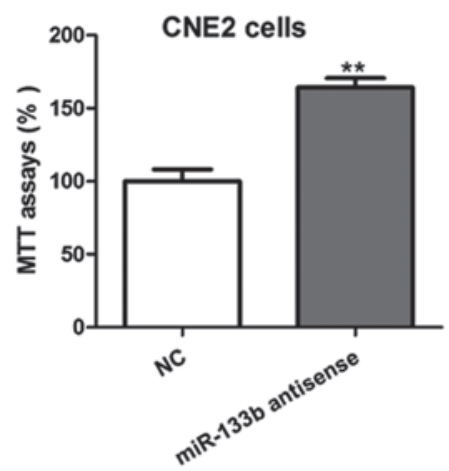

C

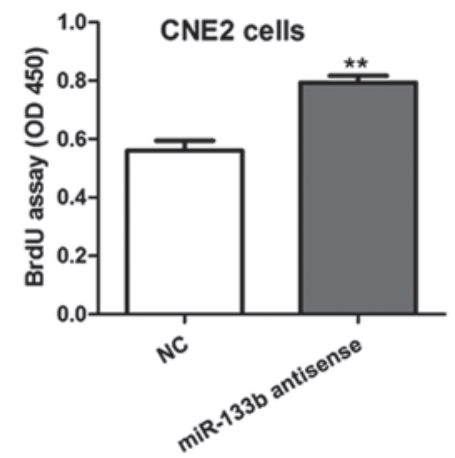

B

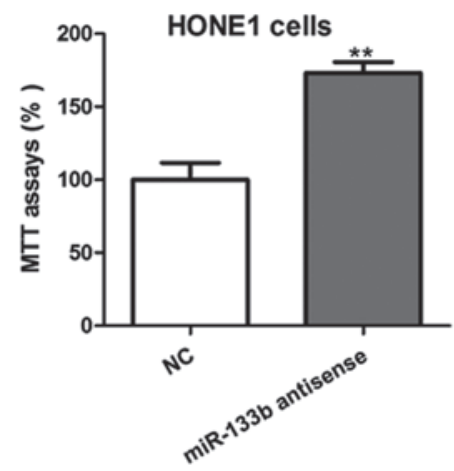

D

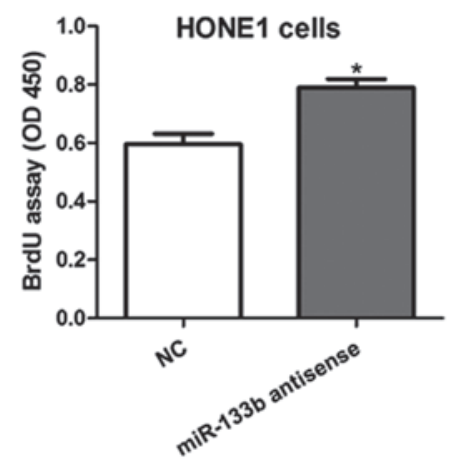

Figure 3. Inhibition of miR-133b promoted the proliferation of NPC cells. The (A and B) cell growth and (C and D) proliferative potentials of CNE2 and HONE1 NPC cells transfected with miR-133b antisense oligonucleotides or NC were determined. The optical density at 450 $\mathrm{nm}$ (OD 450) was assayed following transfection for $24 \mathrm{~h}$. " $\mathrm{P}<0.05$ and ${ }^{* *} \mathrm{P}<0.01$ vs. the NC. miR, microRNA; NPC, nasopharyngeal carcinoma; NC, normal control; MTT, 3-(4,5-dimethylthiazol-2-yl)-2,5-diphenyltetrazolium bromide; BrdU, bromodeoxyuridine.

mouse anti-human glyceraldehyde-3-phosphate dehydrogenase (GAPDH) monoclonal antibody (1:2,000; sc-365062; Santa Cruz Biotechnology). Subsequently, the membrane was washed three times for 5 min each with Tris-buffered saline containing Tween-20 (Beyotime Institute of Biotechnology), prior to incubation with horseradish peroxidase-conjugated mouse 
A

\author{
miR-133b 3'-UUUGGUCCCCUUCAACCAGCUA-5' \\ S1PR1 (WT) 5'-UGCGAGUUACGAUAGGGUCGAU-3' \\ S1PR1 (Mut) 5'-UGCGAGUUACGAUAGGGAAAAU-3'
}

B

CNE2 cells

NC miR-133b mimics

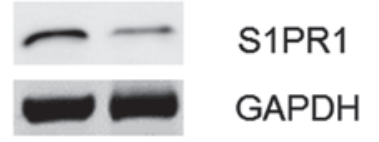

C HONE1 cells
D

$\mathbf{E}$

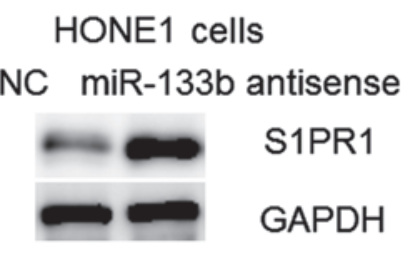

CNE2 cells

NC miR-133b antisense

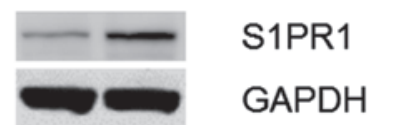

NC miR-133b antisense
S1PR1
GAPDH

$\mathbf{F}$

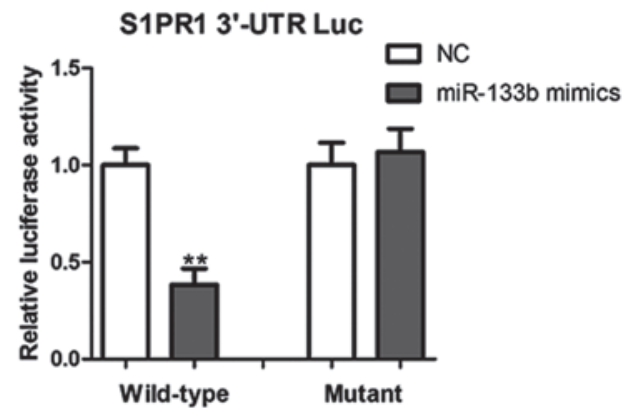

NC miR-133b mimics

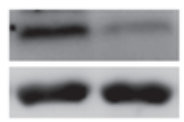

S1PR1

GAPDH
G
Patient \#1

Normal NPC

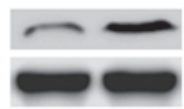

\section{S1PR1 GAPDH}

Patient \#2

Normal NPC

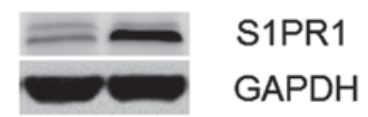

Figure 4. miR-133b repressed S1PR1 protein expression by targeting its 3'-UTR. (A) Prediction of miR-133b binding sites in the 3'-UTRs of the human S1PR1 gene using bioinformatics. A potential binding site is highlighted in bold. Relative protein expression levels of S1PR1 in the (B) CNE2 and (C) HONE1 NPC cells transfected with miR-133b mimics or the NC. Relative protein expression levels of S1PR1 in the (D) CNE2 and (E) HONE1 NPC cells transfected with miR-133b antisense oligonucleotides or the NC. (F) Luciferase reporter assays in CNE2 cells. Cells were transfected with wild-type or mutant 3'-UTR-reporter constructs containing miR-133b mimics or the NC. (G) Representative western blots showing the relative protein expression levels of S1PR1 in NPC tissues and adjacent non-cancerous normal tissues (Normal) from two patients. ${ }^{* *} \mathrm{P}<0.01$ vs. the NC. miR, microRNA; S1PR1, sphingosine-1-phosphate receptor 1; WT, wild type; Mut, mutant; 3'-UTR, 3'-untranslated region; NPC, nasopharyngeal carcinoma; NC, normal control; GAPDH, glyceraldehyde-3-phosphate dehydrogenase.

anti-rabbit IgG (1:2,000; sc-2491; Santa Cruz Biotechnology), Anti-Mouse IgG1 VHH Single Domain Antibody (1:1,000; ab193651; Abcam, Cambridge, UK) and mouse anti-goat IgG (1:2,000; sc-2489; Santa Cruz Biotechnology) for $2 \mathrm{~h}$ at $25^{\circ} \mathrm{C}$. The antibody complexes were detected using the SuperSignal West Pico Chemiluminescent Substrate kit (Pierce Biotechnology), according to manufacturer's protocol. The protein expression levels of S1PR1 and STAT3 were normalized to those of GAPDH, using Quantity One software, version 4.62 (Bio-Rad Laboratories, Inc.).

Luciferase reporter assays. Total cDNA from CNE2 cells was used to amplify the 3'-untranslated region (UTR) of S1PR1 by PCR. The primers used were as follows: Forward, 5'-CTC TGTATGACTCTAATA-3' and reverse, 5'-GACCGTCGATCG ACCT-3'. The S1PR1 3'-UTR was cloned into the Ambion pMIR-REPORT ${ }^{\circledR}$ miRNA Expression Reporter Vector (Thermo Fisher Scientific, Inc.), to yield pMIR-REPORT-S1PR1. Mutations were introduced in potential miR-133b binding sites using the QuikChange II Site-Directed Mutagenesis kit (Agilent Technologies, Inc., Santa Clara, CA, USA). Subsequently, the CNE2 cells were transfected with the pMIR-REPORT vectors containing either the wild-type or mutant S1PR1 3'-UTR, and with either the miR-133b mimic or NC plasmids, using Lipofectamine 2000, according to the manufacturer's protocol. The pRL-TK vector (Promega Corporation), encoding the Renilla luciferase gene, was used as an internal control to normalize the transfection efficiency. Luciferase values were determined using the Dual-Luciferase ${ }^{\circledR}$ Reporter Assay system (Promega Corporation).

Statistical analysis. Data are presented as the mean \pm standard error of the mean of $\geq 3$ separate experiments. Statistical analyses were conducted using SAS software, version 9.2 (SAS Institute Inc., Cary, NC, USA). Differences between groups were analyzed using the Student's t-test. $\mathrm{P}<0.05$ was considered to indicate a statistically significant difference.

\section{Results}

miR-133b expression levels are reduced in NPC tissues. In order to determine the expression levels of miR-133b, RT-qPCR was performed using 23 paired NPC tissues and adjacent normal tissues. The results demonstrated that miR-133b was 
A

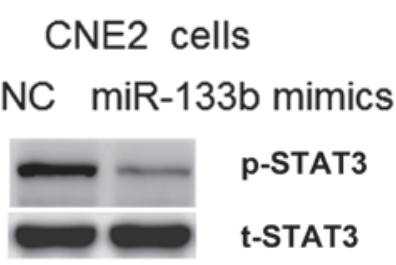

C

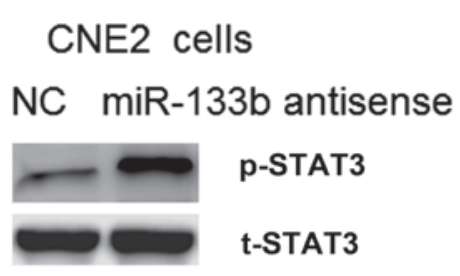

B

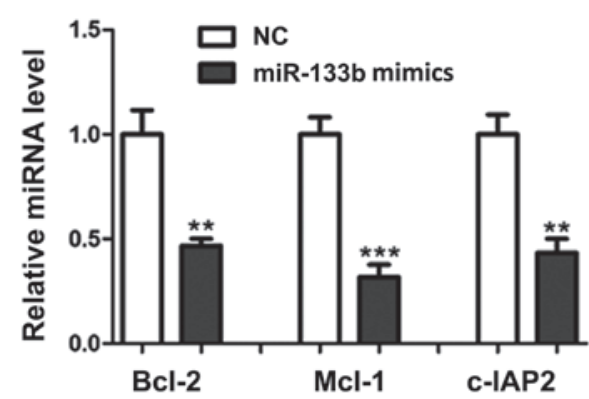

D

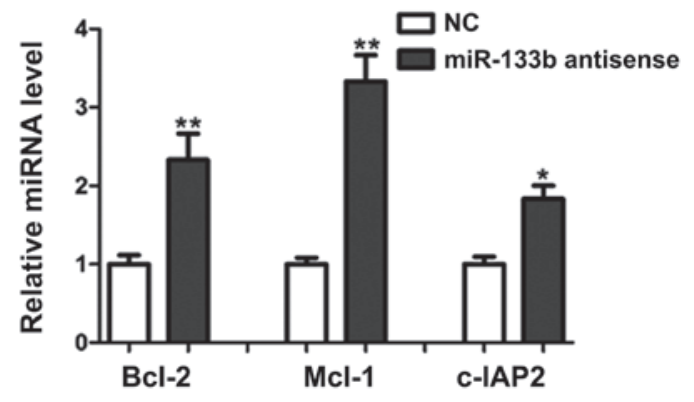

Figure 5. miR-133b regulated STAT3 signaling in NPC cells. (A) Relative protein expression levels of p-STAT3 in CNE2 NPC cells transfected with miR-133b mimics or the NC; t-STAT3 was used as a loading control. (B) mRNA expression levels of Bcl-2, Mcl-1 and c-IAP2 in CNE2 cells transfected with miR-133b mimics or the NC. (C) Relative protein expression levels of p-STAT3 in CNE2 cells transfected with miR-133b antisense oligonucleotides or the NC. (D) mRNA expression levels of Bcl-2, Mcl-1 and c-IAP2 in CNE2 cells transfected with miR-133b antisense oligonucleotides or the NC. ${ }^{*} \mathrm{P}<0.05,{ }^{* *} \mathrm{P}<0.01$ and ${ }^{* * * *} \mathrm{P}<0.001$ vs. the NC. miR, microRNA; STAT3, signal transducer and activator of transcription 3; NPC, nasopharyngeal carcinoma; p-STAT3, phosphorylated-STAT3; t-STAT3, total STAT3; Bcl-2, B-cell lymphoma-2; Mcl-1, myeloid cell leukemia-1; c-IAP2, cellular inhibitor of apoptosis-2; NC, normal control.

significantly downregulated in NPC tissues, as compared with adjacent normal tissues ( $\mathrm{P}<0.01$; Fig. 1).

miR-133b overexpression inhibits NPC cell proliferation. In order to evaluate the biological function of miR-133b in NPC cell proliferation, miR-133b mimics or NC were transfected into CNE2 and HONE1 cells, and MTT and BrdU incorporation assays were conducted. Overexpression of miR-133b in the two cell lines was confirmed using RT-qPCR (Fig. 2A and B). MTT assays demonstrated that cell growth was significantly inhibited by miR-133b overexpression (Fig. 2C and D). Consistent with this, BrdU assays showed that cell proliferation was markedly reduced in cells overexpressing miR-133b, as compared with those transfected with the NC (Fig. 2E and F). Furthermore, the inhibition of endogenous miR-133b by transfection of the CNE2 and HONE1 cells with antisense oligonucleotides was shown to promote the growth and proliferation of the cells (Fig. 3). These results indicate that miR-133b is able to inhibit the proliferation of NPC cells in vitro.

miR-133b inhibits the proliferation of NPC cells via downregulation of SIPR1. Potential direct targets of miR-133b were predicted using miWalk software, which revealed that S1PR1 harbors a potential miR-133b binding site (Fig. 4A). Therefore, RT-qPCR and western blotting assays were conducted in order to investigate the effect of miR-133b on the protein expression levels of S1PR1. Notably, the protein expression levels of S1PR1 were reduced in the cells overexpressing miR-133b (Fig. 4B and C). In addition, the protein expression levels of S1PR1 were markedly increased following inhibition of miR-133b with the antisense oligo- nucleotides (Fig. 4D and E). These results suggest that miR-133b may regulate S1PR1 expression at the translational level.

In order to confirm this, the full-length $3^{\prime}$-UTR of the human S1PR1 gene was cloned into the pMIR-REPORT vector downstream of the luciferase gene. Overexpression of the miR-133b mimics led to a significant reduction in luciferase activity when the reporter construct contained the S1PR1 3'-UTR (Fig. 4F). However, mutation of the miR-133b binding site within the S1PR1 3'-UTR abolished this effect of miR-133b (Fig. 4F). Consistent with this, it was observed that protein expression levels of S1PR1 were upregulated in the NPC tissues, as compared with the adjacent normal tissues (Fig. 4G); thus suggesting that S1PR1 is a direct target of miR-133b in NPC cells.

miR-133b regulates STAT3 signaling in NPC cells. It has previously been shown that S1PR1 is crucial for persistent STAT3 activation in cancer cells (16). Therefore, the present study examined STAT3 signaling pathways in the NPC cells following the overexpression or depletion of miR-133b. The protein expression levels of phosphorylated STAT3 were markedly reduced by miR-133b overexpression (Fig. 5A). Concordantly, the mRNA expression levels of B-cell lymphoma-2, myeloid cell leukemia-1 and cellular inhibitor of apoptosis-2, which are downstream targets of STAT3 signaling, were also inhibited by miR-133b (Fig. 5B). Conversely increased activation of these STAT3 signaling-associated proteins was observed in the cells following the depletion of miR-133b (Fig. 5C and D). These results suggest that miR-133b may regulate the S1PR1-mediated activation of STAT3 signaling. 


\section{Discussion}

In the present study, miR-133b was shown to be expressed at significantly lower levels in NPC samples, as compared with adjacent normal tissue samples. However, the molecular events underlying the downregulation of miR-133b remain unclear. Niederwieser et al (17) reported that high expression levels of DNA (cytosine-5-)-methyltransferase $3 \beta$ were associated with differentially expressed miR-133b in older adults with primary, cytogenetically normal acute myeloid leukemia. Therefore, the present study hypothesized that epigenetic factors may contribute to the downregulation of miR-133b, although this requires further investigation in future studies.

It is well known that each miRNA may regulate multiple gene targets. Previous studies have identified numerous target genes of miR-133b in a variety of tumor types; these genes include TBPL1, fibroblast growth factor receptor-1, specificity protein-1 and epidermal growth factor receptor (11,12,18-20); thus suggesting that the roles of miR-133b in tumorigenesis may be cell- or tissue-specific. The present study demonstrated that miR-133b overexpression inhibited, whereas its suppression increased, endogenous S1PR1 protein expression in NPC cells. Furthermore, luciferase reporter assays further identified S1PR1 as a direct target of miR-133b in NPC cells. Therefore, the results of the present study suggested that miR-133b may inhibit NPC cell proliferation, at least in part, by repressing S1PR1 expression.

Previous studies have reported upregulation of S1PR1, a $\mathrm{G}$ protein-coupled receptor for lysophospholipid sphingosine-1-phosphate (S1P), in STAT3-positive tumors (21-23). S1PR1-mediated STAT3 activation has been shown to occur in part by upregulation of the Janus Kinase 2 tyrosine kinase activity, whereas ablation of S1PR1 in tumor cells or immune cells inhibited tumor STAT3 activity (23). Given that STAT3 has a crucial role in promoting the progression of human cancers $(23,24)$, molecules or chemical compounds that target S1P/S1PR1 may be effective novel therapeutic agents for the prevention of malignant progression. Notably, in the present study, miR-133b was demonstrated to regulate STAT3 activation in NPC cells, further confirming a tumor suppressive role for miR-133b.

In conclusion, the present study demonstrated that miR-133b expression was downregulated in NPC tissues. Furthermore, miR-133b overexpression was able to inhibit the cell proliferation of NPC by reducing the protein expression levels of S1PR1. The results of the present study highlight an important role for miR-133b in NPC progression, which may aid the development of novel therapeutics for the treatment of patients with NPC.

\section{References}

1. Cao SM, Simons MJ and Qian CN: The prevalence and prevention of nasopharyngeal carcinoma in China. Chin J Cancer 30: 114-119, 2011.

2. Coghill AE and Hildesheim A: Epstein-Barr virus antibodies and the risk of associated malignancies: Review of the literature. Am J Epidemiol 180: 687-695, 2014.

3. Stoker SD, van Diessen JN, de Boer JP, Karakullukcu B, Leemans CR and Tan IB: Current treatment options for local residual nasopharyngeal carcinoma. Curr Treat Options Oncol 14: 475-491, 2013.
4. He ML, Luo MX, Lin MC and Kung HF: MicroRNAs: Potential diagnostic markers and therapeutic targets for EBV-associated nasopharyngeal carcinoma. Biochim Biophys Acta 1825: 1-10, 2012.

5. Liu N, Tang LL, Sun Y, Cui RX, Wang HY, Huang BJ, He QM, Jiang W and Ma J: MiR-29c suppresses invasion and metastasis by targeting TIAM1 in nasopharyngeal carcinoma. Cancer Lett 329: 181-188, 2013.

6. Tan JK, Tan EL and Gan SY: Elucidating the roles of miR-372 in cell proliferation and apoptosis of nasopharyngeal carcinoma TW01 cells. Exp Oncol 36: 170-173, 2014.

7. Zhang ZC, Li YY, Wang HY, Fu S, Wang XP, Zeng MS, Zeng YX and Shao JY: Knockdown of miR-214 promotes apoptosis and inhibits cell proliferation in nasopharyngeal carcinoma. PLoS One 9: e86149, 2014.

8. Wong TS, Man OY, Tsang CM, Tsao SW, Tsang RK, Chan JY, Ho WK, Wei WI and To VS: MicroRNA let-7 suppresses nasopharyngeal carcinoma cells proliferation through downregulating c-Myc expression. J Cancer Res Clin Oncol 137: 415-422, 2011.

9. Nohata N, Hanazawa T, Enokida H and Seki N: MicroRNA-1/133a and microRNA-206/133b clusters: Dysregulation and functional roles in human cancers. Oncotarget 3: 9-21, 2012.

10. Zhao Y, Huang J, Zhang L, Qu Y, Li J, Yu B, Yan M, Yu Y, Liu B and Zhu Z: MiR-133b is frequently decreased in gastric cancer and its overexpression reduces the metastatic potential of gastric cancer cells. BMC Cancer 14: 34, 2014

11. Wen D, Li S, Ji F, Cao H, Jiang W, Zhu J and Fang X: MiR-133b acts as a tumor suppressor and negatively regulates FGFR1 in gastric cancer. Tumour Biol 34: 793-803, 2013.

12. Xiang KM and Li XR: MiR-133b acts as a tumor suppressor and negatively regulates TBPL1 in colorectal cancer cells. Asian Pac J Cancer Prev 15: 3767-3772, 2014.

13. Zhao H, Li M, Li L, Yang X, Lan G and Zhang Y: MiR-133b is down-regulated in human osteosarcoma and inhibits osteosarcoma cells proliferation, migration and invasion and promotes apoptosis. PLoS One 8: e83571, 2013.

14. Zhang C, Yao C, Li H, Wang G and He X: Serum levels of microRNA-133b and microRNA-206 expression predict prognosis in patients with osteosarcoma. Int J Clin Exp Pathol 7: 4194-4203, 2014

15. Livak KJ and Schmittgen TD: Analysis of relative gene expression data using real-time quantitative PCR and the $2-\Delta \Delta \mathrm{Ct}$ method. Methods 25: 402-408, 2001.

16. Priceman SJ, Shen S, Wang L, Deng J, Yue C, Kujawski M and $\mathrm{Yu} \mathrm{H}$ : S1PR1 is crucial for accumulation of regulatory $\mathrm{T}$ cells in tumors via STAT3. Cell Rep 6: 992-999, 2014.

17. Niederwieser C, Kohlschmidt J, Volinia S, Whitman SP, Metzeler KH, Eisfeld AK, Maharry K, Yan P, Frankhouser D, Becker H, et al: Prognostic and biologic significance of DNMT3B expression in older patients with cytogenetically normal primary acute myeloid leukemia. Leukemia 29: 567-575, 2015.

18. Qiu T, Zhou X, Wang J, Du Y, Xu J, Huang Z, Zhu W, Shu Y and Liu P: MiR-145, miR-133a and miR-133b inhibit proliferation, migration, invasion and cell cycle progression via targeting transcription factor Sp1 in gastric cancer. FEBS Lett 588: 1168-1177, 2014.

19. Liu L, Shao X, Gao W, Zhang Z, Liu P, Wang R, Huang P, Yin Y and Shu Y: MicroRNA-133b inhibits the growth of non-small-cell lung cancer by targeting the epidermal growth factor receptor. FEBS J 279: 3800-3812, 2012.

20. Tao J, Wu D, Xu B, Qian W, Li P, Lu Q, Yin C and Zhang W: MicroRNA-133 inhibits cell proliferation, migration and invasion in prostate cancer cells by targeting the epidermal growth factor receptor. Oncol Rep 27: 1967-1975, 2012.

21. Liang J, Nagahashi M, Kim EY, Harikumar KB, Yamada A, Huang WC, Hait NC, Allegood JC, Price MM, Avni D, et al: Sphingosine-1-phosphate links persistent STAT3 activation, chronic intestinal inflammation, and development of colitis-associated cancer. Cancer Cell 23: 107-120, 2013.

22. Deng J, Liu Y, Lee H, Herrmann A, Zhang W, Zhang C, Shen S, Priceman SJ, Kujawski M, Pal SK, et al: S1PR1-STAT3 signaling is crucial for myeloid cell colonization at future metastatic sites. Cancer Cell 21: 642-654, 2012.

23. Lee H, Deng J, Kujawski M, Yang C, Liu Y, Herrmann A, Kortylewski M, Horne D, Somlo G, Forman S, et al: STAT3-induced S1PR1 expression is crucial for persistent STAT3 activation in tumors. Nat Med 16: 1421-1428, 2010.

24. Yu H, Lee H, Herrmann A, Buettner R and Jove R: Revisiting STAT3 signalling in cancer: New and unexpected biological functions. Nat Rev Cancer 14: 736-746, 2014. 